\title{
Fast Implementation of Oversampled Modulated Filter Banks
}

\section{Stephan Weiss}

\author{
Dept. of Electronics \& Computer Science \\ University of Southampton \\ Southampton SO17 1BJ, UK \\ s.weissdecs.soton.ac.uk
}

\author{
Robert W. Stewart \\ Dept. of Electrical \& Electronic Engineering \\ University of Strathclyde \\ Glasgow G1 1XW, Scotland, UK \\ r.stewart@eee.strath.ac.uk
}

\begin{abstract}
This paper presents an efficient implementation of oversampled filter banks derived from a prototype filter by modulation. Via a polyphase analysis, redundancies in the filter operations are removed. With some modifications, a very simple and efficient implementation is found, which is briefly compared to existing realisations.
\end{abstract}

\section{INTRODUCTION}

Oversampled filter banks are widely used for reducing the computational complexity of resourcedemanding signal processing algorithms, such as subband adaptive filtering applied to acoustic echo control $[4,1]$. Therefore, low complexity realisations of such filter banks are desirable. However, despite this motivation and in contrast to their critically decimated counterparts [6], numerically efficient implementations of non-critically (or "oversampled") filter banks have received little attention.

The sparse literature on the implementation of oversampled filter banks includes the work of Wackersreuther [7], where a time domain approach leads to a factorisation of the analysis filter bank operation into a filtering operation linked to the prototype filter coefficients, a cyclical shift, and the applications of the appropriate modulating transform (e.g. a DFT). In [5], the analysis filter bank is in the time-domain divided into a filter operation with time-varying components of the prototype filter, followed by the modulating transform. More recently, polyphase factorisations in the $\mathrm{z}$-domain have been presented [2,8]. For all cases [7, 5, 2, 8], a dual implementation can be found for the synthesis filter bank operation.

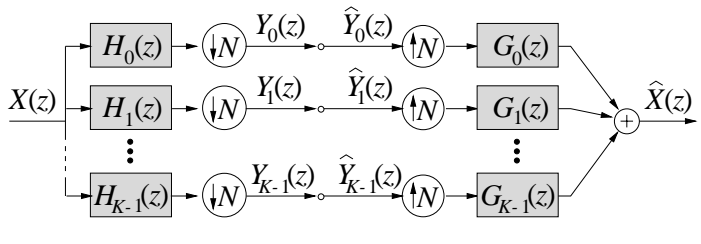

Fig. 1: Analysis and synthesis filter bank for subband decomposition of a signal $X(z)$.

analysis filter bank synthesis filter bank

Here, the polyphase approach $[2,8]$ is utilised as a starting point to yield, with some modifications and rearrangements, novel, simple and efficient filter bank implementations.

\section{ANALYSIS FILTER BANK}

Consider the analysis filter bank of Fig. 1 producing $K$ subband signals. To remove computational redundancies due to the decimation by $N$, a type-1 polyphase notation [6] is introduced for the analysis filters,

$$
H_{k}(z)=\sum_{n=0}^{N-1} z^{-n} H_{k \mid n}(z)
$$

The input signal is decomposed into $N$ type-2 polyphase components,

$$
X(z)=\sum_{n=0}^{N-1} z^{-N+n-1} X_{n}(z)
$$


Organising the polyphase components in vector form,

$$
\begin{aligned}
\underline{H}_{k}(z) & =\left[\begin{array}{llll}
H_{k \mid 0}(z) & H_{k \mid 1}(z) & \cdots & H_{k \mid N-1}(z)
\end{array}\right]^{\mathrm{T}} \\
\underline{X}(z) & =\left[\begin{array}{llll}
X_{0}(z) & X_{1}(z) & \cdots & X_{N-1}(z)
\end{array}\right]^{\mathrm{T}}
\end{aligned}
$$

the $K$ subband signals $\underline{Y}(z)=\left[Y_{0}(z) Y_{1}(z) \cdots Y_{K-1}(z)\right]^{\mathrm{T}}$ can be denoted by

$$
\underline{Y}(z)=\left[\underline{H}_{0}(z) \underline{H}_{1}(z) \cdots \underline{H}_{K-1}(z)\right]^{\mathrm{T}} \cdot \underline{X}(z)=\mathbf{H}(z) \cdot \underline{X}(z)
$$

where $\mathbf{H}(z)$ is the polyphase analysis matrix [2] and describes a linear periodically time-varying system of period $N$.

We assume that the analysis filters are FIR with $L_{p}$ coefficients, $h_{k, 0} \cdots h_{k, L_{p}-1}$, which are derived from a prototype lowpass filter by a modulation sequence $t_{k, 0} \cdots t_{k, L_{p}-1}$ with period $K$. For simplicity (w.l.o.g.) we assume that $L_{p}$ is a common multiple of both $N$ and $K$. With $\mathbf{I}_{N}$ being an $N \times N$ identity matrix and the filter coefficients of $H_{k}(z)$ organised in a vector $\mathbf{h}_{k}$, the polyphase components in (3) can be written as

$$
\begin{aligned}
& \underline{H}_{k}(z)=\underbrace{\left[\begin{array}{llll}
\mathbf{I}_{N} & z^{-1} \cdot \mathbf{I}_{N} & \cdots & z^{-L_{p} / N+1} \cdot \mathbf{I}_{N}
\end{array}\right]}_{\mathbf{L}_{1}^{\mathrm{T}}(z)} \cdot \mathbf{h}_{k} \\
& =\mathbf{L}_{1}^{\mathrm{T}}(z) \cdot \underbrace{\left[\begin{array}{cccc}
p_{0} & & & \mathbf{0} \\
& p_{1} & & \\
& & \ddots & \\
\mathbf{0} & & & p_{L_{p}-1}
\end{array}\right]}_{\mathbf{P}} \cdot \underbrace{\left[\begin{array}{c}
t_{k, 0} \\
t_{k, 1} \\
\vdots \\
t_{k, L_{p}-1}
\end{array}\right]}_{\mathbf{t}_{k}}
\end{aligned}
$$

where $\mathbf{t}_{k} \in \mathbb{C}^{L_{p}}$ is the modulation sequence for the $k$ th analysis filter. The periodicity of this sequence can be exploited by

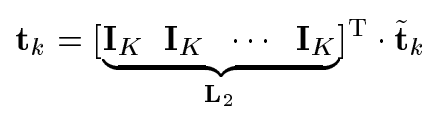

where $\tilde{\mathbf{t}}_{k} \in \mathbb{C}^{K}$. The $K$ modulation sequences are collected in a matrix

$$
\mathbf{T}=\left[\begin{array}{lll}
\tilde{\mathbf{t}}_{0} & \cdots & \tilde{\mathbf{t}}_{L_{p}-1}
\end{array}\right]^{\mathrm{T}}
$$

which for example for a DFT modulated filter bank would be a $K \times K$ DFT matrix. The polyphase analysis matrix is now given by

$$
\mathbf{H}(z)=\mathbf{T} \cdot \mathbf{L}_{2} \cdot \mathbf{P} \cdot \mathbf{L}_{1}(z)
$$

Hence, a factorisation into prototype filter components and a rotation by a transform matrix $\mathbf{T}$ has been established similar to $[2,8]$. The difference is that the diagonal matrix $\mathbf{P}$ contains no sparse filters but only the prototype filter coefficients.

\section{SYNTHESIS FILTER BANK}

The synthesis filter bank with expansion by $N$ followed by interpolation filters $G_{k}(z)$ as shown in Fig. 1 can be performed in an analogous fashion to the analysis filter bank operation. The condition that all filters $G_{k}(z)$ and $H_{k}(z)$ are derived from the same prototype lowpass filter and that the filter bank is perfectly reconstructing is guaranteed by $\mathbf{H}(z)$ being paraunitary [2]. Reconstruction is then given by the polyphase synthesis matrix

$$
\mathbf{G}(z)=\mathbf{H}^{\mathrm{H}}\left(z^{-1}\right)=\mathbf{L}_{1}^{\mathrm{T}}\left(z^{-1}\right) \cdot \mathbf{P} \cdot \mathbf{L}_{2}^{\mathrm{T}} \cdot \mathbf{T}^{\mathrm{H}}
$$

This polyphase synthesis matrix relates the subband samples back to the polyphase components of the fullband signal, $\underline{\hat{X}}(z)=\mathbf{G}(z) \cdot \underline{\hat{Y}}(z)$. 


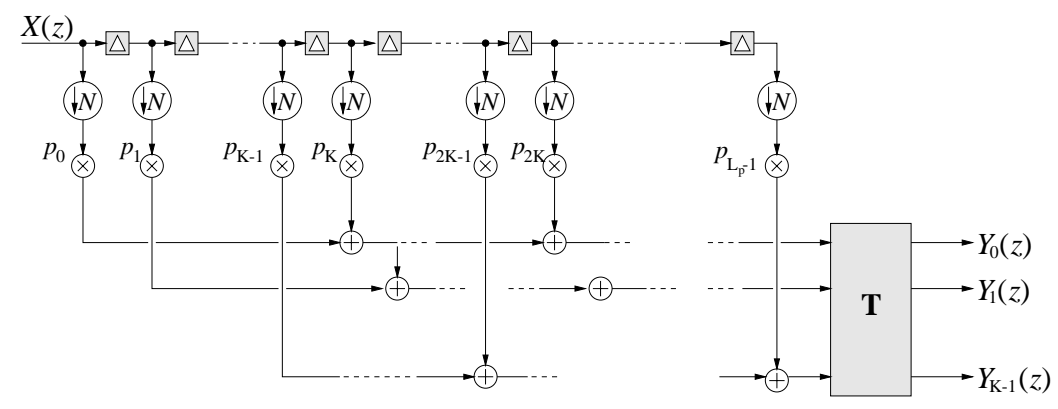

Fig. 2: Analysis filter bank signal flow graph.

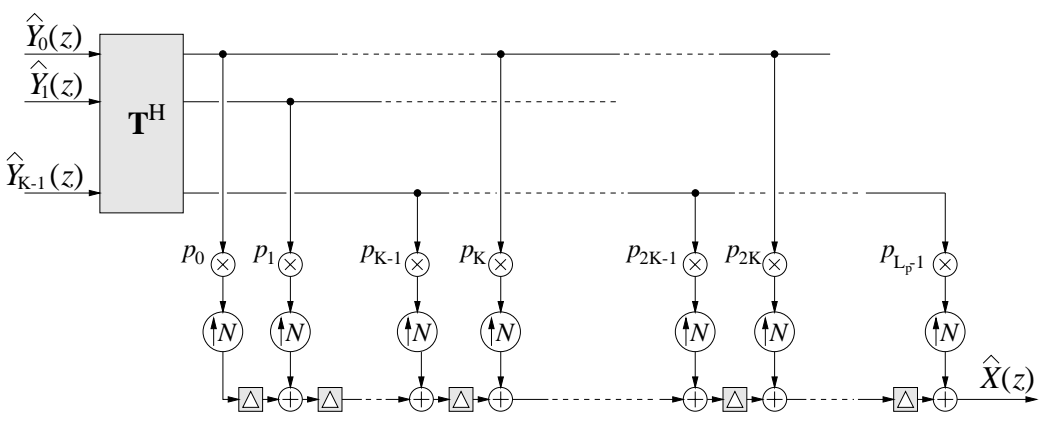

Fig. 3: Synthesis filter bank signal flow graph.

\section{IMPLEMENTATION}

The analysis filter bank operation in (10) with can be executed in two steps. First, the memoryrequiring multiplication $\mathbf{L}_{N}(z) \cdot \underline{X}(z)$ can be brought into the form of a tapped delay line (TDL) block updated with $N$ new samples for every operation. This is shown in Fig. 2. The second part of the operation is memoryless, and consists of the multiplication with the $L_{p}$ prototype filter coefficients, forming $K$ polyphase components, which are then rotated by the modulation matrix $\mathbf{T}$ to yield the subband samples.

For the synthesis filter bank operations, we split the equation $\underline{X}(z)=\mathbf{G}(z) \cdot \underline{Y}(z)$ into two parts by introducing an intermediate variable $\underline{V}(z) \in \mathbb{C}^{L_{p}}(z)$ :

$$
\underline{V}(z)=\left[\begin{array}{cccc}
\mathbf{0}_{N} & & & \mathbf{0} \\
\mathbf{I}_{N} & \mathbf{0}_{N} & & \\
& \ddots & \ddots & \\
\mathbf{0} & & \mathbf{I}_{N} & \mathbf{0}_{N}
\end{array}\right] \cdot \underline{V}(z) z^{-1}+\mathbf{P} \cdot \mathbf{L}_{2}^{\mathrm{T}} \cdot \mathbf{T}^{\mathrm{H}} \cdot \underline{Y}(z)
$$

The second summand on the right hand side of (12) is memory-less, and the system matrix in (12) performs a shift operation by $N$ samples [3]. The desired output can be derived from the intermediate variable $\underline{V}(z)$ as

$$
\underline{X}(z)=\left[\begin{array}{llll}
\mathbf{0} & \cdots & \mathbf{0} & \mathbf{I}_{N}
\end{array}\right] \cdot \underline{V}(z)
$$

Thus, the only memory-requiring operation in the synthesis filter bank is the shift operation in (12), and the circuit given in Fig. 3 results. This circuit shows the de-rotation of the subband samples by $\mathbf{T}^{\mathrm{H}}$, the copying by $\mathbf{L}_{2}$ such that the $L_{p}$ multipliers are excited, and accumulation of the products into a TDL. This TDL results from rearranging the multiplexing of the $N$ polyphase outputs in $\underline{X}(z)$, and only requires a shift operation every $N$ sampling periods. The algorithms for both analysis and synthesis filter bank operations are given in Tab. 1.

\section{COMPUTATIONAL COMPLEXITY}

From the signal flow graphs for analysis and synthesis in Figs. 2 and 3, the computational complexities for both operations in terms of multiply-accumulates (MACs) evaluations is

$$
C=\frac{1}{N} \cdot\left(2 L_{p}+4 K \log _{2} K\right) \quad[\mathrm{MACs}],
$$




\begin{tabular}{|c||c|}
\hline \multicolumn{2}{|c|}{ Analysis Filter Bank } \\
\hline \hline 1: & update $\underline{x}$ with $N$ new samples \\
2: & repeat for $k=0(1) K-1:$ \\
& $\hat{x}_{k}=\underline{x}(k: K: \text { end })^{T} \cdot \underline{p}(k: K:$ end $)$ \\
3: & $\underline{y}=\mathbf{T} \cdot \underline{\hat{x}}$ \\
\hline
\end{tabular}

\begin{tabular}{|c||c|}
\hline \multicolumn{2}{|c|}{ Synthesis Filter Bank } \\
\hline \hline $1:$ & $\underline{\hat{x}}=\mathbf{T}^{\mathrm{H}} \cdot \underline{y}$ \\
$2:$ & repeat for $\underline{l}=0(1) L_{p}-1:$ \\
& $v(l)=v(l)+p(l) \cdot \hat{x}\left(\bmod _{K} l\right)$ \\
3: & the last $N$ samples in $\underline{v}$ are outputs samples \\
4: & shift $\underline{v}$ by $N$ samples, padding with zeros. \\
\hline
\end{tabular}

Tab. 1: Algorithms for analysis (top) and synthesis filter bank (bottom); arrays are indicated as underlined quantities, and their elements are marked by indices (subscripts or bracketed). Both filter bank routines have to be executed only once within a sampling period at the low subband rate.

for a complex input $x[n]$. Multiplication of the complex samples with the real valued prototype filter coefficients accrues to $2 L_{p}$ MACs. The modulation matrix $\mathbf{T}$ is assumed to be implemented by a $K$-point FFT [8] requiring $4 K \log _{2} K$ real valued MACs. If the fullband signal $X(z)$ is real valued, the complexity $C$ in (14) is halved since all MACs outwith the transform are entirely real valued and half of the subband signals $Y_{k}(z)$ are complex conjugate copies of others subbands, and therefore do not need to be generated nor processed.

Although other methods reported in the literature give identical complexities in terms of MACs, the realisations in Figs. 2 and 3 do not require any additional circular shifts [7], or the indexing of time-varying filters [5] or filters with sparse coefficients [2, 8].

\section{CONCLUSION}

A filter bank analysis has been presented based on the polyphase approach. With some modifications, filter bank realisations have been derived which are efficient, very simple, and avoid some of the disadvantages of previous fast implementations.

\section{REFERENCES}

[1] C. Breining, P. Dreiseitel, E. Hänsler, A. Mader, B. Nitsch, H. Puder, T. Schertler, G. Schmidt, and J. Tilp. "Acoustic Echo Control". IEEE Signal Processing Magazine, Vol.16(No.4):pp.1999, July 1999.

[2] Z. Cvetković and M. Vetterli. "Tight Weyl-Heisenberg Frames in $l^{2}(\mathbb{Z})$ ". IEEE Transactions on Signal Processing, Vol.46(No.5):pp.1256-1259, May 1998.

[3] G. H. Golub and C. F. Van Loan. Matrix Computations. Johns Hopkins University Press, Baltimore, Maryland, 3rd edition, 1996.

[4] W. Kellermann. "Analysis and Design of Multirate Systems for Cancellation of Acoustical Echoes". In Proc. IEEE International Conference on Acoustics, Speech, and Signal Processing, volume 5, pages 2570-2573, New York, 1988.

[5] R. Stasinski. "Efficient Implementation of Uniform Filter Banks in the Absence of Critical Sampling”. IEE Electronics Letters, Vol.30(No.2):pp.118-1120, January 1994.

[6] P. P. Vaidyanathan. Multirate Systems and Filter Banks. Prentice Hall, Englewood Cliffs, 1993.

[7] G. Wackersreuther. Ein Beitrag zum Entwurf digitaler Filterbänke. PhD thesis, Insitut für Nachrichtentechnik, Universität Erlangen-Nürnberg, 1987.

[8] S. Weiss. "On Adaptive Filtering in Oversampled Subbands". PhD thesis, Signal Processing Division, University of Strathclyde, Glasgow, May 1998. 